\title{
ljfis
}

\section{On Soft $w$-Structures Defined by Soft Sets}

\section{Won Keun Min}

Department of Mathematics, Kangwon National University, Chuncheon, Korea

\begin{abstract}
In this work, we introduce the notion of soft $w$-structure and investigate some basic properties of this new structure by using the concept of soft set. Moreover, we study the notions of soft $w-T_{0}$ (soft $w-T_{1}$, soft $w-T_{2}$ ).
\end{abstract}

Keywords: Soft set, Soft topology, Soft $w$-structure, Soft $w-T_{0}\left(w-T_{1}, w-T_{2}\right)$.

\section{Introduction and Preliminaries}

In 1999, Molodtsov [1] initiated the notion of soft set theory as a new mathematical tool which is free from the complex problems. Later on Maji et al. [2] proposed several operations on soft sets and some basic properties and then Pei and Miao [3] investigated the relationships between soft sets and information systems.

In 2011, Shabir and Naz [4] introduced the notion of soft topological spaces and the author [5] corrected some their results. Zorlutuna et al. [6] continued to study the properties of soft topological spaces by defining the concepts of interior and soft neighborhoods in soft topological spaces. In 2011, Cagman et al. [7] defined soft topological spaces by modifying the soft set. Also, Roy and Samanta [8] strengthen the definition of the soft topological spaces presented in [7].

In 2017, with the aim of generalizing the notion of soft topology, Zakari et al. [9] introduced a soft weak structure. Recently, Al-Saadi and Min [10] investigated the notion of soft generalized closed sets in a soft weak structure.

Meanwhile, Min and Kim [11] introduced a new notion called weak structures as the following: Let $X$ be a non-empty set and $P(X)$ be its power set. A structure $\omega$ on $X$ is called

Received: Oct. 15, 2018

Revised : Apr. 10, 2020

Accepted: Apr. 23, 2020

Correspondence to: Won Keun Min (wkmin@kangwon.ac.kr)

(CThe Korean Institute of Intelligent Systems

(c) This is an Open Access article distributed under the terms of the Creative Commons Attribution Non-Commercial License (http://creativecommons.org/licenses/ by-nc/3.0// which permits unrestricted noncommercial use, distribution, and reproduction in any medium, provided the original work is properly cited. a weak structure on $X$ if and only if (i) $\phi \in \omega$ : (ii) For $U, V \in \omega, U \cap V \in \omega$.

In this work, by applying the notion of weak structure in [11], we want to introduce the new notion of soft $w$-structure which is a generalized soft topological structure defined by Shabir and Naz [10] and a stronger structure than soft weak structure defined by Zakari et al. [9]. And we investigate some basic properties of this new class by using the notion of soft set. Moreover, we study the notions of soft $w-T_{0}$ (soft $w-T_{1}$, soft $w-T_{2}$ ) and some properties of such notions. From now on, let $X$ be a non-empty common universe, $E$ a set of parameters, and $P(X)$ denote the power set of $X$.

Definition 1.1 ( [1]). For $A \subseteq E$, a pair $(F, A)$ is called a soft set over $X$, where $F$ is a mapping given by $F: A \rightarrow P(X)$. For $e \in A, F(e)$ may be considered as the set of $e$-approximate elements of the soft set $(F, A)$.

Definition $1.2([2])$. A soft set $(F, A)$ over $X$ is said to be: 
1) A null soft set denoted by $\tilde{\emptyset}$ if $F(e)=\emptyset$ for all $e \in A$.

2) An absolute soft set denoted by $\tilde{X}$ if $F(e)=X$ for all $e \in A$.

Definition 1.3 ( [2]). For any two soft sets $(F, A)$ and $(G, B)$ defined over a common universe $X$, we have:

1) $(F, A) \tilde{\subset}(G, B)$ iff $A \subseteq B$ and $F(e) \subseteq G(e)$ for all $e \in A$.

2) $(F, A)=(G, B)$ iff $(F, A) \tilde{\subset}(G, B)$ and $(G, B) \tilde{\subset}(F, A)$.

3) $(F, A) \tilde{\cup}(G, B)=(H, C)$ where $C=A \cup B$ and

$$
H(e)= \begin{cases}F(e), & \text { if } e \in A-B, \\ G(e), & \text { if } e \in B-A, \\ F(e) \cup G(e), & \text { if } e \in A \cap B,\end{cases}
$$

for all $e \in C$.

4) $(F, A) \tilde{\cap}(G, B)=(K, D)$ where $D=A \cap B$ and $K(e)=$ $F(e) \cap G(e)$ for all $e \in C$.

5) $x \in(F, A)$ where $x \in X$ iff $x \in F(e)$ for all $e \in A$ and $x \notin(F, A)$ whenever $x \notin F(e)$ for some $e \in A$.

Definition $1.4([12])$. For a soft set $(F, A)$ over $X$, the relative complement of $(F, A)$ (denoted by $\left.(F, A)^{\prime}\right)$ is defined by $(F, A)^{\prime}=\left(F^{\prime}, A\right)$, where $F^{\prime}: A \rightarrow P(X)$ is given by $F^{\prime}(e)=X-F(e)$ for all $e \in A$.

Definition 1.5 ( [4]). Let $\tau$ be the collection of soft sets over $X$. Then $\tau$ is called a soft topology on $X$ if $\tau$ satisfies the following axioms:

1) $\tilde{\emptyset}, \tilde{X}$ belong to $\tau$.

2) The union of any number of soft sets in $\tau$ belong to $\tau$.

3) The intersection of any two soft sets in $\tau$ belong to $\tau$.

The triple $(X, \tau, E)$ is called a soft topological space over $X$. The member of $\tau$ are said to be soft open in $X$. A soft set $(F, E)$ over $X$ is said to be soft closedin $X$ if its relative complement $(F, E)^{\prime}$ belong to $\tau$.

\section{2. $\quad$ Soft $w$-Structures}

Definition 2.1. Let $s w$ be the collection of soft sets over $X$. Then $s w$ is called $a$ soft $w$-structure on $X$ if $s w$ satisfies the following axioms:

- $\tilde{\emptyset}, \tilde{X}$ belong to $s w$.
- The intersection of any two soft sets in $s w$ belongs to $s w$.

The triple $(X, s w, E)$ is called a soft $w$-space over $X$. The member of $s w$ is said to be soft w-open in $X$. A soft set $(F, E)$ over $X$ is said to be soft $w$-closed in $X$ if its relative complement $(F, E)^{\prime}$ belongs to $s w$.

Remark 2.2. Let $s w$ be a soft $w$-structure over $X$. The soft $w$-structure $s w$ is a kind of generalized soft topology and a stronger structure than a soft weak structure defined by Zakari et al. [9] as the following: Let $X$ be a non-empty set and $E$ a set of parameters. A collection $\omega$ of soft sets defined over $X$ with respect to $E$ is called a soft weak structure $[9]$ iff $\tilde{\emptyset} \in \omega$.

Example 2.3. Let $X=\left\{h_{1}, h_{2}, h_{3}, h_{4}\right\}, E=\left\{e_{1}, e_{2}\right\}$ and $s w=\left\{\tilde{\emptyset}, \tilde{X},\left(F_{1}, E\right),\left(F_{2}, E\right),\left(F_{3}, E\right)\right\}$, where

$$
\begin{array}{ll}
F_{1}\left(e_{1}\right)=\left\{h_{2}, h_{3}\right\}, & F_{2}\left(e_{2}\right)=\left\{h_{1}, h_{2}\right\} ; \\
F_{2}\left(e_{1}\right)=\left\{h_{1}, h_{2}\right\}, & F_{3}\left(e_{2}\right)=\left\{h_{1}, h_{3}\right\} ; \\
F_{3}\left(e_{1}\right)=\left\{h_{2}\right\}, & F_{4}\left(e_{2}\right)=\left\{h_{1}\right\} .
\end{array}
$$

Then $s w$ is a soft $w$-structure over $X$ with respect to $E$ but not a soft topology.

Definition 2.4. Let $s w$ be a soft $w$-structure over $X$ with respect to $E$. For a soft set $(F, E)$ over $X$, the soft $w$-closure of $(F, E)$ (simply, $\left.c_{s w}(F, E)\right)$ and the soft $w$-interior of $(F, E)$ (simply, $\left.c_{s w}(F, E)\right)$ are defined as the following:

$$
\begin{aligned}
& \text { - } i_{s w}(F, E)=\tilde{\cup}\{(G, E):(G, E) \tilde{\subset}(F, E),(G, E) \in s w\} . \\
& \text { - } c_{s w}(F, E)=\tilde{\cap}\left\{(H, E):(F, E) \tilde{\subset}(H, E),(H, E)^{\prime} \in s w\right\} .
\end{aligned}
$$

Theorem 2.5. Let $s w$ be a soft $w$-structure over $X$ with respect to the parameters set $E$ and $(F, E)$ a soft set. If there exists a soft $w$-open set $(G, E)$ such that $x \in(G, E) \tilde{\subset}(F, E)$, then $x \in i_{s w}(F, E)$

Proof. It is obvious.

Example 2.6. As in Example 2.3, consider the soft $w$-structure sw over $X$ with respect to $E$ and a soft set $\left(F_{4}, E\right)$ as follows:

$$
F_{4}\left(e_{1}\right)=\left\{h_{1}, h_{2}, h_{3}\right\}, \quad F_{4}\left(e_{2}\right)=\left\{h_{1}, h_{2}, h_{3}\right\} .
$$

Then $\left(F_{4}, E\right)=i_{s w}\left(F_{4}, E\right)$. For $h_{3} \in i_{s w}\left(F_{4}, E\right)$, there is no a soft $w$-open set containing $h_{3}$ in $s w$. So the converse of Theorem 2.5 is not always true.

Theorem 2.7. Let $s w$ be a soft $w$-structure over $X$ with respect to the parameters set $E$ and $(F, E)$ a soft set. If $x \in c_{s w}(F, E)$, 
then $(G, E) \tilde{\cap}(F, E) \neq \tilde{\emptyset}$ for all $(G, E) \in s w$ such that $x \in$ $(G, E)$.

Proof. Let $x \in c_{s w}(F, E)$. Suppose that there exists an element $(G, E) \in s w$ such that $x \in(G, E)$ and $(F, E) \tilde{\cap}(G, E)=$ $\tilde{\emptyset}$. Then $(F, E) \subset(G, E)^{\prime}$, so $c_{s w}(F, E) \tilde{\subset}(G, E)^{\prime}$ and $x \notin$ $c_{s w}(F, E)$. So it is a contradiction.

Example 2.8. Let $X=\left\{h_{1}, h_{2}, h_{3}\right\}, E=\left\{e_{1}, e_{2}\right\}$ and $s w=$ $\left\{\tilde{\emptyset}, \tilde{X},\left(F_{1}, E\right),\left(F_{2}, E\right),\left(F_{3}, E\right)\right\}$ where

$$
\begin{array}{ll}
F_{1}\left(e_{1}\right)=\left\{h_{2}, h_{3}\right\}, & F_{2}\left(e_{2}\right)=\left\{h_{1}, h_{2}\right\} ; \\
F_{2}\left(e_{1}\right)=\left\{h_{1}, h_{2}\right\}, & F_{3}\left(e_{2}\right)=\left\{h_{1}, h_{3}\right\} ; \\
F_{3}\left(e_{1}\right)=\left\{h_{2}\right\}, & F_{4}\left(e_{2}\right)=\left\{h_{1}\right\} .
\end{array}
$$

Then $s w$ is a soft $w$-structure over $X$ with respect to $E$. Consider a soft set $\left(F_{4}, E\right)$ defined as:

$$
F_{4}\left(e_{1}\right)=\left\{h_{1}\right\}, \quad F_{4}\left(e_{2}\right)=\left\{h_{3}\right\} .
$$

Since $\left(F_{4}, E\right)$ is soft $w$-closed, $\left(F_{4}, E\right)=c_{s w}\left(F_{4}, E\right)$. For $h_{1} \in X,\left(F_{2}, E\right)$ is the only soft $w$-open set and $\left(F_{4}, E\right) \tilde{\cap}\left(F_{2}, E\right)$ $\neq \tilde{\emptyset}$, however, $h_{1} \tilde{\notin} c_{s w}\left(F_{4}, E\right)$. So the converse of Theorem 2.7 is not always true.

Theorem 2.9. Let $s w$ be a soft $w$-structure defined over $X$ with respect to the parameters set $E$ and $(F, E)$ be a soft set.

- If $(F, E)$ is a soft $w$-open set, then $(F, E)=i_{s w}(F, E)$.

- If $(F, E)$ is a soft $w$-closed set, then $(F, E)=c_{s w}(F, E)$.

Proof. From the definitions of soft $w$-interior and soft $w$-closure, it is obvious.

But the converses in Theorem 2.9 are not always true as shown the next example.

Example 2.10. Let $X=\left\{h_{1}, h_{2}, h_{3}\right\}, E=\left\{e_{1}, e_{2}\right\}$ and $s w=\left\{\tilde{\emptyset}, \tilde{X},\left(F_{1}, E\right),\left(F_{2}, E\right),\left(F_{3}, E\right),\left(F_{4}, E\right),\left(F_{5}, E\right)\right\}$, where

$$
\begin{array}{ll}
F_{1}\left(e_{1}\right)=\left\{h_{3}\right\}, & F_{1}\left(e_{2}\right)=\left\{h_{2}\right\} ; \\
F_{2}\left(e_{1}\right)=\left\{h_{2}, h_{3}\right\}, & F_{2}\left(e_{2}\right)=\left\{h_{1}, h_{2}\right\} ; \\
F_{3}\left(e_{1}\right)=\left\{h_{1}, h_{2}\right\}, & F_{3}\left(e_{2}\right)=\left\{h_{1}, h_{3}\right\} ; \\
F_{4}\left(e_{1}\right)=\left\{h_{1}\right\}, & F_{4}\left(e_{2}\right)=\left\{h_{3}\right\} ; \\
F_{5}\left(e_{1}\right)=\left\{h_{2}\right\}, & F_{4}\left(e_{2}\right)=\left\{h_{1}\right\} ; \\
F_{6}\left(e_{1}\right)=\left\{h_{1}, h_{3}\right\}, & F_{4}\left(e_{2}\right)=\left\{h_{2}, h_{3}\right\} ;
\end{array}
$$

Then $s w$ is a soft $w$-structure over $X$ with respect to $E$. For a soft set $\left(F_{5}, E\right), c_{s w}\left(F_{5}, E\right)=\left(F_{5}, E\right)$ but $\left(F_{5}, E\right)$ is not soft $w$-closed. And, for a soft set $\left(F_{6}, E\right), i_{s w}\left(F_{6}, E\right)=\left(F_{6}, E\right)$ but $\left(F_{6}, E\right)$ is not soft $w$-open.

Theorem 2.11. Let $s w$ be a soft $w$-structure over $X$ with respect to $E$. Let $(F, E)$ and $(G, E)$ be two soft sets over $X$. Then:

- $i_{s w}(F, E) \tilde{C}(F, E)$.

- If $(F, E) \tilde{C}(G, E)$, then $i_{s w}(F, E) \tilde{\subset} i_{s w}(G, E)$.

- $i_{s w}((F, E) \tilde{\cap}(G, E))=i_{s w}(F, E) \tilde{\cap} i_{s w}(G, E)$.

- $i_{s w}\left(i_{s w}(F, E)\right)=i_{s w}(F, E)$

Proof. (1) and (2) are obvious.

(3) It is obvious that $i_{s w}((F, E) \tilde{\cap}(G, E)) \tilde{\complement} i_{s w}(F, E) \tilde{\cap} i_{s w}$ $(G, E)$ from (2). For soft $w$-open sets $(U, E) \tilde{C}(F, E)$ and $(V, E) \tilde{C}(G, E),(U, E) \tilde{\cap}(V, E)$ is a soft $w$-open set contained in $(F, E) \tilde{\cap}(G, E)$. This implies that

$$
i_{s w}(F, E) \tilde{\cap} i_{s w}(G, E) \tilde{\complement} i_{s w}((F, E) \tilde{\cap}(G, E)) .
$$

(4) From (1), it follows $i_{s w}\left(i_{s w}(F, E)\right) \tilde{\subseteq} i_{s w}(F, E)$. For any soft $w$-open set $(U, E)$ such that $(U, E) \tilde{\subseteq} i_{s w}(F, E),(U, E)=$ $i_{s w}(U, E) \tilde{\simeq} i_{s w}\left(i_{s w}(F, E)\right)$, and so $i_{s w}(F, E) \tilde{\tilde{\subseteq}} i_{s w}\left(i_{s w}(F, E)\right)$. Consequently, we have $i_{s w}\left(i_{s w}(F, E)\right)=i_{s w}(F, E)$.

Theorem 2.12. Let $s w$ be a soft $w$-structure defined over $X$ with respect to $E$. If $(F, E)$ and $(G, E)$ are two soft sets over $X$, then:

- $(F, E) \tilde{C} c_{s w}(F, E)$.

- If $(F, E) \tilde{C}(G, E)$, then $c_{s w}(F, E) \tilde{\complement} c_{s w}(G, E)$.

- $c_{s w}(F, E) \tilde{\cup} c_{s w}(G, E)=c_{s w}((F, E) \tilde{\cup}(G, E))$.

- $c_{s w}\left(c_{s w}(F, E)\right)=c_{s w}(G, E)$.

- $i_{s w}(F, E)^{\prime}=\left(c_{s w}(F, E)\right)^{\prime}$ and $c_{s w}(F, E)^{\prime}=\left(i_{s w}(F, E)\right)^{\prime}$.

Proof. It is similar to the proof of Theorem 2.11.

Now, we introduce the separation axioms in soft $w$-space with a soft $w$-structure $s w$.

Definition 2.13. Let $s w$ be a soft $w$-structure over $X$ with respect to $E$. A soft $w$-space $(X, s w, E)$ is called: 
- $w-T_{0}$ if for each $x, y \in X$ such that $x \neq y$, there exists a soft $w$-open set $(F, E)$ such that $x \in(F, E)$ and $y \notin(F, E)$ or $x \notin(F, E)$ and $y \in(F, E)$.

- $w-T_{1}$ if for each $x, y \in X$ such that $x \neq y$, there exist soft $w$-open sets $(F, E)$ and $(G, E)$ such that $x \in(F, E)$ and $y \notin(F, E)$ and $x \notin(G, E)$ and $y \in(G, E)$.

- $w-T_{2}$ if for each $x, y \in X$ such that $x \neq y$, there exist soft $w$-open sets $(F, E)$ and $(G, E)$ such that $x \in(F, E)$, $y \in(G, E)$ and $(F, E) \tilde{\cap}(G, E)=\tilde{\emptyset}$.

We have the following diagram:

$$
\text { soft } w-T_{2} \Rightarrow \text { soft } w-T_{1} \Rightarrow \text { soft } w-T_{0} \text {. }
$$

Example 2.14. Let $X=\left\{h_{1}, h_{2}, h_{3}\right\}, E=\left\{e_{1}, e_{2}\right\}$ and $s w=\left\{\tilde{\emptyset}, \tilde{X},\left(F_{1}, E\right),\left(F_{2}, E\right),\left(F_{3}, E\right),\left(F_{4}, E\right),\left(F_{5}, E\right)\right.$, $\left.\left(F_{6}, E\right)\right\}$, where

$$
\begin{array}{ll}
F_{1}\left(e_{1}\right)=\left\{h_{3}\right\}, & F_{1}\left(e_{2}\right)=\left\{h_{3}\right\} ; \\
F_{2}\left(e_{1}\right)=\left\{h_{1}, h_{2}\right\}, & F_{3}\left(e_{2}\right)=\left\{h_{1}, h_{3}\right\} ; \\
F_{3}\left(e_{1}\right)=\left\{h_{2}, h_{3}\right\}, & F_{2}\left(e_{2}\right)=\left\{h_{1}, h_{2}\right\} ; \\
F_{4}\left(e_{1}\right)=\left\{h_{2}\right\}, & F_{4}\left(e_{2}\right)=\left\{h_{1}\right\} ; \\
F_{5}\left(e_{1}\right)=\left\{h_{3}\right\}, & F_{4}\left(e_{2}\right)=\emptyset ; \\
F_{6}\left(e_{1}\right)=\emptyset, & F_{4}\left(e_{2}\right)=\left\{h_{3}\right\} .
\end{array}
$$

Then $s w$ is a soft $w$-structure over $X$ with respect to $E$. It is obviously a soft $w-T_{1}$ space. For $h_{1}, h_{2} \in X,\left(F_{2}, E\right)$ and $\left(F_{3}, E\right)$ are unique soft $w$-open sets of $h_{1}, h_{2}$, respectively. But $\left(F_{2}, E\right) \tilde{\cap}\left(F_{3}, E\right) \neq \tilde{\emptyset}$. So $(X, s w, E)$ is not soft $w-T_{2}$.

Example 2.15. Let $X=\left\{h_{1}, h_{2}, h_{3}\right\}, E=\left\{e_{1}, e_{2}\right\}$ and $s w=\left\{\tilde{\emptyset}, \tilde{X},\left(F_{1}, E\right),\left(F_{2}, E\right),\left(F_{3}, E\right),\left(F_{4}, E\right)\right\}$, where

$$
\begin{array}{ll}
F_{1}\left(e_{1}\right)=\left\{h_{1}\right\}, & F_{1}\left(e_{2}\right)=\left\{h_{1}\right\} ; \\
F_{2}\left(e_{1}\right)=\left\{h_{2}\right\}, & F_{3}\left(e_{2}\right)=\left\{h_{2}\right\} ; \\
F_{3}\left(e_{1}\right)=\left\{h_{1}, h_{3}\right\}, & F_{4}\left(e_{2}\right)=X ; \\
F_{4}\left(e_{1}\right)=\emptyset, & F_{4}\left(e_{2}\right)=\left\{h_{2}\right\} .
\end{array}
$$

Then $s w$ is a soft $w$-structure over $X$ with respect to $E$. It is obviously a soft $w-T_{0}$ space but it is not soft $w-T_{1}$.

Let $s w$ be a soft $w$-structure over $X$ with respect to $E$. A soft $w$-space $(X, s w, E)$ is called relative soft $w-T_{0}$ if for each $x, y \in X$ such that $x \neq y$, there exists a soft $w$-open set $(F, E)$ such that $x \in(F, E)$ and $y \in(F, E)^{\prime}$ or $x \in(F, E)^{\prime}$ and $y \in(F, E)$.
Theorem 2.16. Let $s w$ be a soft $w$-structure on $X$. If $X$ is a relative soft $w-T_{0}$ space, then for each $x, y \in X$ such that $x \neq y$, we have $c_{s w}(x, E) \neq c_{s w}(y, E)$.

Proof. Let $X$ be a relative soft $w-T_{0}$ and $x, y \in X$ such that $x \neq y$. Then there exists a soft $w$-open set $(F, E)$ such that $x \in(F, E)$ and $y \in(F, E)^{\prime}$. Therefore $(F, E)^{\prime}$ is a soft $w$-closed set such that $x \notin(F, E)^{\prime}$ and $y \in(F, E)^{\prime}$. Since $c_{s w}(y, E)$ is the intersection of all soft $w$-closed subsets containing $(y, E), c_{s w}(y, E) \tilde{C}(F, E)^{\prime}$ and hence $x \notin c_{s w}(y, E)$. Thus $c_{s w}(x, E) \neq c_{s w}(y, E)$.

Theorem 2.17. Let $s w$ be a soft $w$-structure on $X$. If $y \in$ $c_{s w}(x, E)$, then for each soft $w$-open set $(G, E)$ containing $y$, there exists a parameter $e \in E$ such that $x \in G(e)$.

Proof. Let $y \in\left(c_{s w}(x, E)\right)$. Then by Theorem 2.7, $(G, E) \tilde{\cap}$ $(x, E) \neq \tilde{\emptyset}$ for all $(G, E) \in s w$ such that $y \in(G, E)$. Since $(G, E) \tilde{\cap}(x, E) \neq \tilde{\emptyset}$, there exists a parameter $e \in E$ such that $x \in G(e)$.

Theorem 2.18. Let $s w$ be a soft weak structure on $X$. A soft $w$-space $(X, s w, E)$ is soft $w-T_{1}$ if $(x, E)$ is soft $w$-closed set for all $x \in X$.

Proof. Let $x, y \in X$ such that $x \neq y$. Then $(x, E)^{\prime}$ and $(y, E)^{\prime}$ are soft $w$-open sets such $y \in(x, E)^{\prime}, x \in(x, E)^{\prime}$ and $y \notin$ $(y, E)^{\prime}, x \in(y, E)^{\prime}$. Hence $X$ is soft $w-T_{1}$.

\section{Conclusions}

The author introduced the notion of soft $w$-structure and investigated some basic properties of this new structure. In the next research, the author will introduce the associated soft $w$ structures induced by soft topologies and study the relationship between soft $w$-structures and associated soft $w$-structure induced by soft topologies.

\section{Conflict of Interest}

No potential conflict of interest relevant to this article was reported.

\section{References}

[1] D. Molodtsov, "Soft set theory—First results," Computers \& Mathematics with Applications, vol. 37, no. 4-5, pp. 19 31, 1999. https://doi.org/10.1016/S0898-1221(99)000565 
[2] P. K. Maji, R. Biswas, and A. R. Roy, "Soft set theory," Computers \& Mathematics with Applications, vol. 45, no. 4-5, pp. 555-562, 2003. https://doi.org/10.1016/S08981221(03)00016-6

[3] D. Pie and D. Miao, "From soft sets to information systems," in Proceedings of 2005 IEEE International Conference on Granular Computing, Beijing, China, 2005, pp. 617-621. https://doi.org/10.1109/GRC.2005.1547365

[4] M. Shabir and M. Naz, "On soft topological spaces," Computers \& Mathematics with Applications, vol. 61, no. 7, pp. 1786-1799, 2011. https://doi.org/10.1016/j.camwa.2011. 02.006

[5] W. K. Min, "A note on soft topological spaces," Computers \& Mathematics with Applications, vol. 62, no. 9, pp. 35243528, 2011. https://doi.org/10.1016/j.camwa.2011.08.068

[6] I. Zorlutuna, M. Akdag, W. K. Min, and S. Atmaca, "Remarks on soft topological spaces," Annals of Fuzzy Mathematics and Informatics, vol. 3, no. 2, pp. 171-185, 2012.

[7] N. Cagman, S. Karatas, and S. Enginoglu, "Soft topology," Computers \& Mathematics with Applications, vol. 62, no. 1, pp. 351-358, 2011. https://doi.org/10.1016/j.camwa. 2011.05 .016

[8] S. Roy and T. K. Samanta, "An introduction of a soft topological spaces," in Proceedings of UGC Sponsored National Seminar on Recent Trends in Fuzzy Set Theory, Rough Set Theory and Soft Set Theory, Howrah, India, 2011, pp. 9-12.
[9] A. H. Zakari, A. Ghareeb, and S. Omran, "On soft weak structures," Soft Computing, vol. 21, no. 10, pp. 25532559, 2017. https://doi.org/10.1007/s00500-016-2136-8

[10] H. S. Al-Saadi and W. K. Min, "On soft generalized closed sets in a soft topological space with a soft weak structure," International Journal of Fuzzy Logic and Intelligent Systems, vol. 17, no. 4, pp. 323-328, 2017. https://doi.org/10.5391/IJFIS.2017.17.4.323

[11] Y. K. Kim and W. K. Min, "On weak structures and wspaces," Far East Journal of Mathematical Sciences, vol. 97, no. 5, pp. 549-561, 2015. http://dx.doi.org/10.17654/ FJMSJul2015_549_561

[12] M. I. Ali, F. Feng, X. Liu, W. K. Min, and M. Shabir, "On some new operations in soft set theory," Computers \& Mathematics with Applications, vol. 57, no. 9, pp. 15471553, 2009. https://doi.org/10.1016/j.camwa.2008.11.009

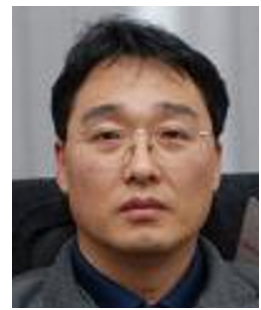

Won Keun Min received the M.S. and the Ph.D. degrees in mathematics from Korea University, Seoul, Korea in 1983 and 1987 , respectively. He is currently a professor in the Department of Mathematics, Kangwon National University. His research interests include general topology, fuzzy topology and soft set theory.

E-mail: wkmin@kangwon.ac.kr 